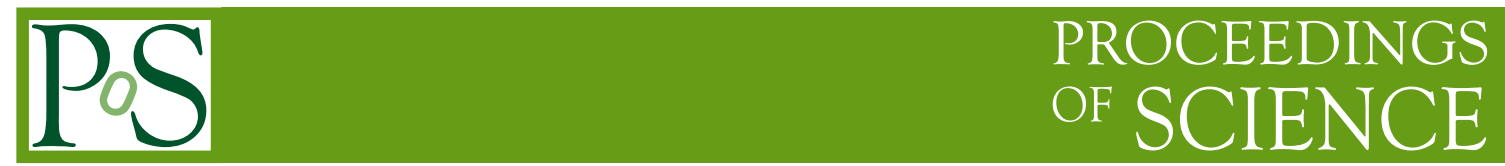

\title{
Majorana "spin-flip" and ultra-low temperature atomic physics
}

\section{Massimo Inguscio*}

LENS and Dipartimento di Fisica, Università di Firenze

Via Nello Carrara, 1 - 50019 Sesto Fiorentino (FI) - Italy

E-mail: ingusciodlens.unifi.it

\begin{abstract}
Atomic gases have been cooled down to ultralow temperatures and Bose-Einstein condensation has been produced thanks to a full control of the "spin-flip" physics investigated by Majorana sixty years earlier [1]. The impact of that seminal work in modern atomic physics and spectroscopy is illustrated.
\end{abstract}

Ettore Majorana's legacy and the Physics of the XXI century October 5-6, 2006

Catania, Italy

\footnotetext{
* Speaker.
} 

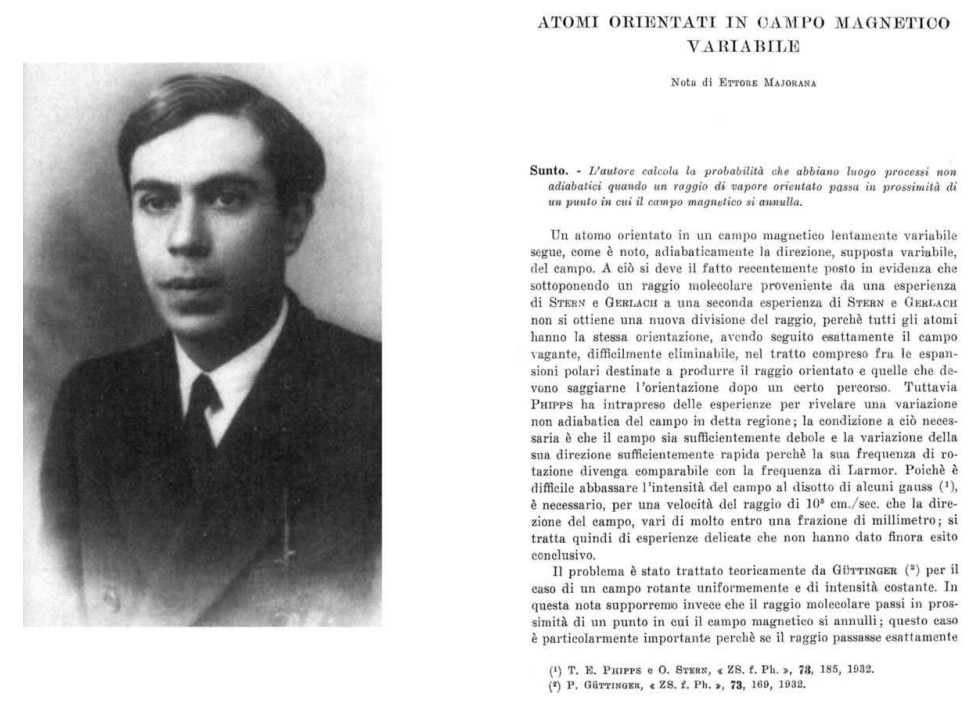

Figure 1: Ettore Majorana and the first page of his 1932 article.

\section{The "spin-flip problem"}

Despite his short scientific activity, Ettore Majorana endowed physics with prominent contributions, not only for his discoveries in the newborn physics of the nuclei, but also for his studies in atomic physics. One of his most influential works in the latter field is represented by the article "Atomi orientati in un campo magnetico variabile" ("Oriented atoms in a variable magnetic field"), published on the Italian journal Nuovo Cimento [1]. This paper, besides having a large impact on the contemporary research on the spatial quantization, still finds important applications in the field of atomic spectroscopy, neutron physics and Bose-Einstein condensation of neutral atoms.

In this work Majorana evaluates the probability of spin-flip transitions for a polarized beam of atoms in the presence of a rapidly varying magnetic field. The problem that Majorana considers "particularly important" is what happens close to a zero of the magnetic field: "all the atoms would invert their spin orientation". The problem had been proposed to Majorana by E. Segré, as E. Amaldi recalls [2]. Indeed E. Segré and R. Frisch [3] were setting up an experiment to generalize the famous Stern and Gerlach works on spatial quantization, that were performed with static magnetic gradients and in adiabatic conditions. In those experiments an atomic beam, after passing through an inhomogeneous magnetic field, was split in two polarized beams, each of the two beams, however, were not split again by a second passing through the same device. This happened because the field gradients were sufficiently weak to ensure the adiabaticity of the process with respect to the internal state of the atoms. The goal of the new experiment was to measure the magnetic moment of an atomic beam, initially prepared in a definite state, after interaction with a rapidly varying magnetic field, where the process was supposed to be non-adiabatic. Differently from similar experiments performed by P. Güttinger [ \], who considered time-dependent fields, in the Segré and Frisch apparatus the non-adiabatic transitions were induced by forcing the atomic beam to travel in a region where a strongly inhomogeneous magnetic field was present, in particular close to a point in which the field was zero. The scheme of the measurement is illustrated in Fig. 2 . 


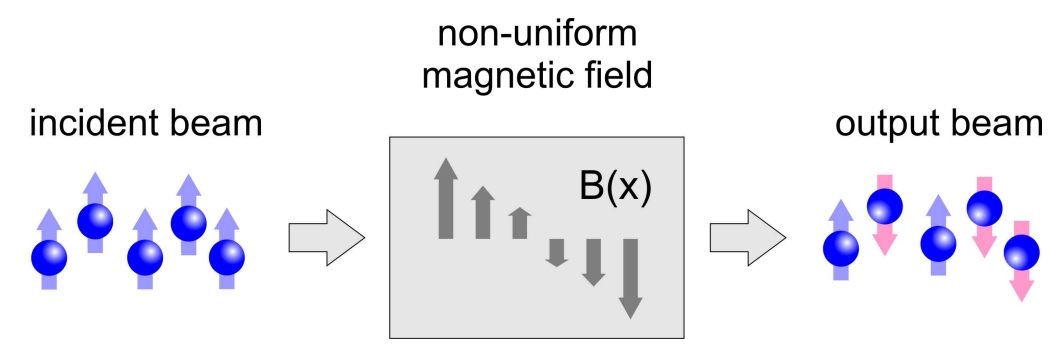

Figure 2: Spin-flip transition of atoms moving in a rapidly varying magnetic field.

In his paper Majorana demonstrates that the effect of a varying magnetic field on an object with angular momentum number $J$ and component $m$ along a quantization axis can be interpreted as a sudden rotation of the angular momentum itself. Majorana develops an elegant formalism for the solution of the problem and finds the exact expression of the rotation angle by a rigorous solution of the time-dependent Schrödinger equation. This angle depends on the magnetic field and the gyromagnetic factor, but not on the initial angular momentum state. As a consequence of this rotation, after interaction with the varying field the system is not in a well defined quantum state with respect to the original field direction, but has to be described as a wavepacket composed by a superposition of states with different $m^{\prime}$. In the paper the probability amplitude for a transition between $m$ and $m^{\prime}$ is explicitly calculated in the simplest case $J=1 / 2$.

Majorana immediately singles out two characteristic frequencies of the problem. The first is the Larmor frequency of the atomic dipole moment $\omega_{L}=g m B / \hbar$. The second is the rotation frequency of the magnetic field in the rest frame of the atom $\omega_{B}$. When the latter frequency becomes larger than the former - this meaning that the field rotates faster than the precession of the atomic dipole around it - the atom has a high probability of changing its magnetic substate, i.e. of undergoing a spin-flip. Majorana demonstrates that the spin-flip probability $P$ depends on the ratio $k=\omega_{L} / \omega_{B}$ between the two frequencies and is given by $P=\exp (-k \pi / 2)$.

As E. Amaldi points out, "the problem was really solved by Majorana with extreme elegance and conciseness for the case $J=1 / 2$ ". The generalization to the case of arbitrary $J$ was completed

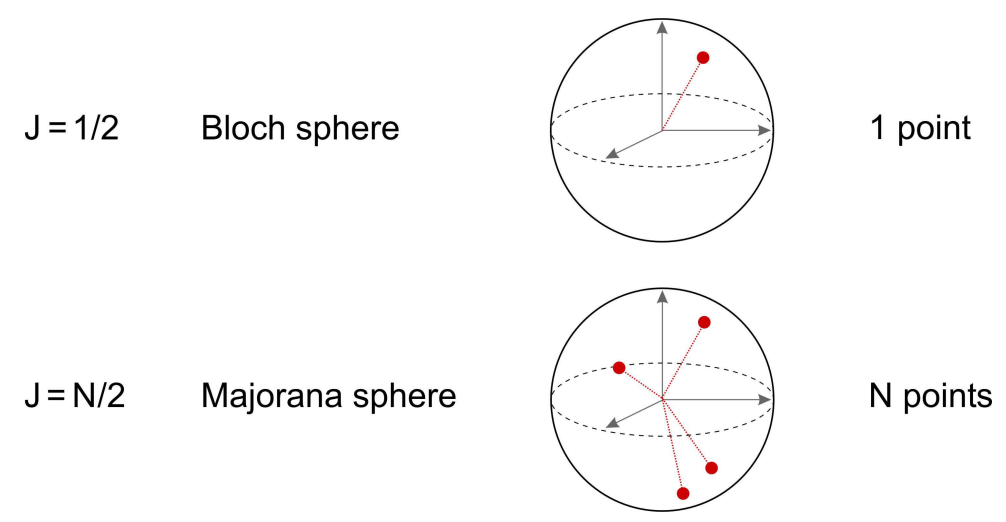

Figure 3: Majorana sphere for the representation of a $J=N / 2$ angular momentum. 

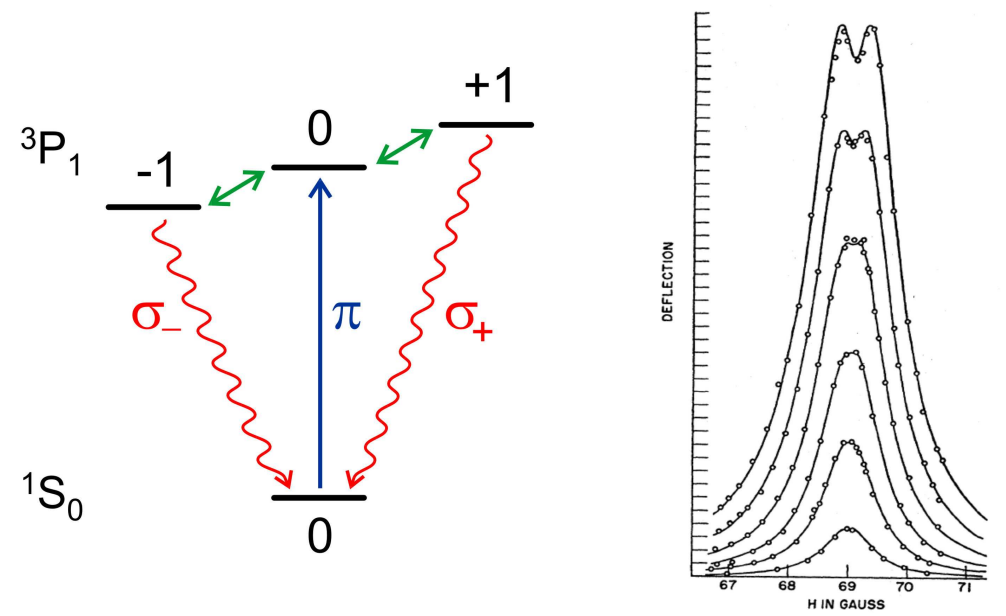

Figure 4: Left) Energy levels of mercury isotopes with even mass and scheme of the double optical + radiofrequency excitation. Right) Spectra obtained by J. Brossel and F. Bitter for different intensities of the radiofrequency excitation (taken from [8]).

by F. Bloch e I. I. Rabi who, in their celebrated work of 1945 [5], cite the work of Majorana as a paper that "has greatly deepened our understanding of the process involved". Indeed, the theoretical basis for the solution of the general problem were already contained in the 1932 paper, where Majorana introduces a novel way to graphically represent a state of angular momentum with arbitrary $J$. In his representation, nowadays known as "Majorana sphere" (or "extended Bloch sphere"), an angular momentum state with $J=N / 2$ is represented by $N$ points on the unit sphere, as sketched in Fig. 3. The rotation of the angular momentum after interaction with the spatially varying field corresponds to a rigid rotation of the $N$ representative points on the Majorana sphere.

Majorana's treatment for spin-flips is used in quantum mechanics textbooks [6] and has recently been rediscovered in atomic and molecular collision physics at low energy [ד]. It is also widely applied outside atomic and molecular physics, e.g. to manipulate polarized beams in neutron physics. Below we discuss two experimental fields were Majorana's work has found an application and is still quite useful: double-resonance spectroscopy and Bose-Einstein condensation.

\section{Double-resonance spectroscopy}

In the early 1950s atomic physicists started to develop double-resonance spectroscopy as a technique to investigate atomic structures. In these experiments atoms were doubly excited by an optical electromagnetic wave, stimulating transitions between different electronic levels, and a radiofrequency (RF) field, inducing transitions between different magnetic substates. In a famous experiment, J. Brossel and F. Bitter [8] studied the polarization of the fluorescence emitted from mercury in the excited ${ }^{3} P_{1}$ level (see Fig. 凹). The atoms, initially in the ${ }^{1} S_{0}$ ground state, were excited with linearly polarized light in order to populate only the sublevel $m=0$ of the ${ }^{3} P_{1}$ state. After the optical excitation a radiofrequency (RF) field was used to induce transitions between $m=0$ and $m= \pm 1$, having different energies owing to the presence of a static magnetic field that removed the degeneracy. Decay of atoms from the states $m= \pm 1$ resulted in the emission 


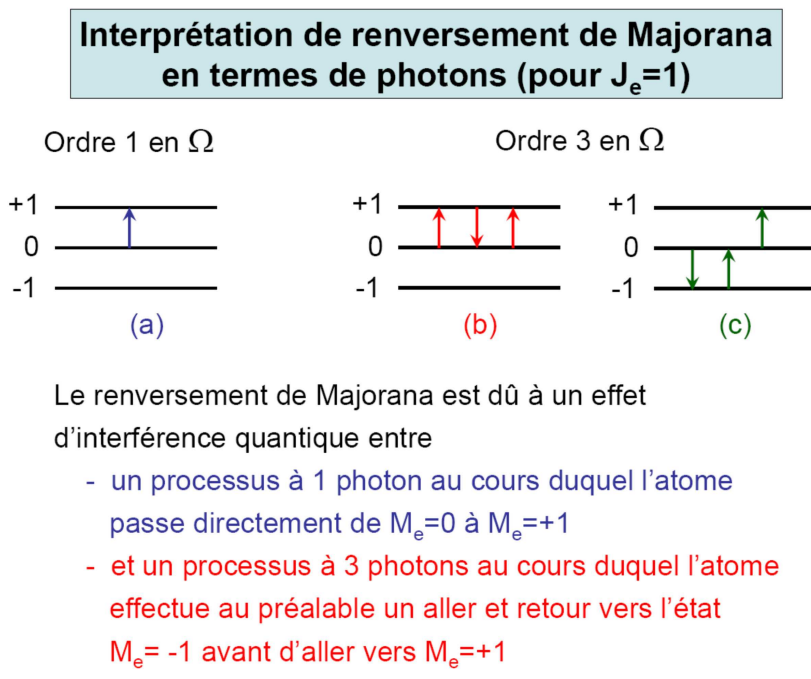

Figure 5: Interpretation of the double-resonance splitting as quantum interference of the direct processes (a) and (b) (including linear excitation and power broadening) with the three-photon process (c) (taken from the 2003-2004 course of Claude Cohen-Tannoudji at the Collège de France [9]).

of circularly polarized light, whose detection provided a measurement of the RF excitation. The observed lineshape of this fluorescence light had a peculiar behaviour, that is shown in the right of Fig. 4: while for weak excitation one observes a curve with only one maximum, when increasing the radiofrequency power the line develops a double-peaked structure with an evident central dip.

In their paper [8] Brossel and Bitter interpret such a behaviour as "Majorana transitions" and use Majorana's formula to perfectly explain the experimental data. Indeed, the RF transitions can be treated as a reorientation of the atomic magnetic dipole $J=1$ in the presence of the rotating RF magnetic field, with the transition probability depending on the field intensity.

The explanation of this experiment still attracts a great interest. In particular, it has been reconsidered by C. Cohen-Tannoudji in his course at Collège de France in 2003 [9]. By developing a fully quantum treatment, he interprets the splitting in the lineshape (nowadays known as "Majorana-Brossel effect") as a quantum interference effect between the linear excitation and higher-order processes. When the strength of the driving field is large, the single-photon excitation from $m=0$ to $m=+1$ can interfere with a three-photon process where the atoms from sublevel $m=0$, before making the transition to $m=+1$, undergo a transition to and from sublevel $m=-1$. This latter process is indeed responsible for the observed dip in the lineshape, called by Cohen-Tannoudji as "renversement de Majorana" (i.e. "Majorana inversion"), as shown in his slide reproduced in Fig. 国.

\section{Magnetic trapping and ultralow-temperature atomic physics}

Coming back to the spin-flip of atoms moving close to a vanishing magnetic field, it is worth noting that the indepth understanding of this effect had been a necessary step for the experimental achievement of Bose-Einstein condensation in atomic gases. In order to observe condensation, 
a) quadrupole trap
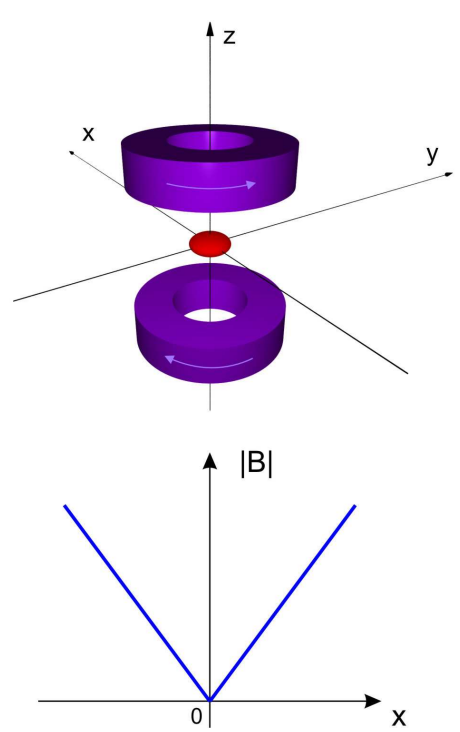

b) "Ioffe-Pritchard" trap
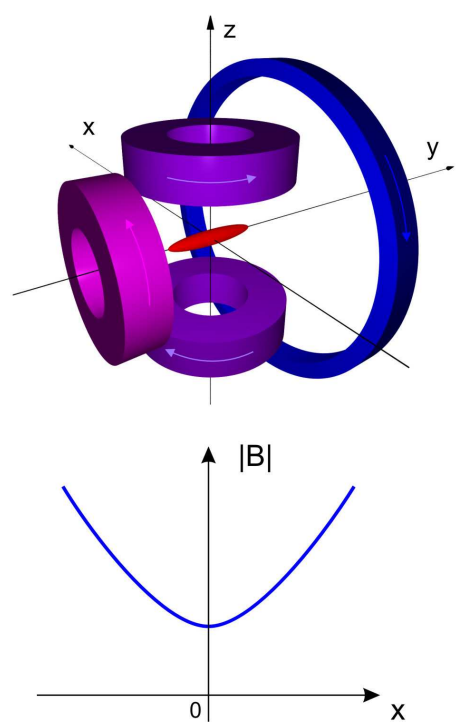

Figure 6: Magnetic traps schemes. a) Two coils in anti-Helmoltz configuration produce a uniform magnetic gradient, i.e. a quadrupole trap centered in the point in which the field is zero. b) By using additional coils one obtains the Ioffe-Pritchard configuration, in which the magnetic field varies harmonically around a non-zero minimum, thus suppressing Majorana spin-flip.

atomic samples must be cooled down to temperatures as low as fractions of a microkelvin. These extremely low temperatures have been achieved by using magnetic traps for atoms pre-cooled with laser radiation and then spin-polarized. The final cooling towards condensation is performed by forced evaporation of the hottest atoms from the magnetic trap and by the consequent thermalization (at a lower temperature) of the remaining atoms via atom-atom collisions.

The magnetic trap in which the atoms are stored is created by a static inhomogeneous magnetic field. Trapping is possible because of the interaction energy of the atomic magnetic dipole moment $\mu$ with the magnetic field $B(\mathbf{r})$, which results in a conservative potential $U=\mu|B(\mathbf{r})|$, provided that the magnetic moment stays adiabatically aligned with the spatially varying magnetic field. The first attempts to achieve condensation were done by using quadrupole magnetic traps, as the one sketched in Fig. 6a, i.e. the same configuration that Majorana has studied in his paper. As the temperature of the sample gets lower the atoms move closer to the trap center, in which the magnetic field is zero, and non-adiabatic spin-flip transitions may take place. When the atoms undergo a spinflip they cease to be confined (since the potential becomes anti-trapping) and Majorana's formula allows a quantitative analysis of the atom losses in terms of the magnetic field gradient and of the atomic temperature. The zero in the magnetic field is a sort of "hole" that reduces the atomic density thus preventing the evaporative cooling, that needs comparatively large densities to achieve rethermalization.

E. A. Cornell, C. E. Wieman and W. Ketterle, who have first realized Bose-Einstein condensation in 1995, were perfectly aware of the problem. Indeed in their Nobel Lectures [10, 11] they often mention the "Majorana hole". The problem that had to be solved was the design of traps with 


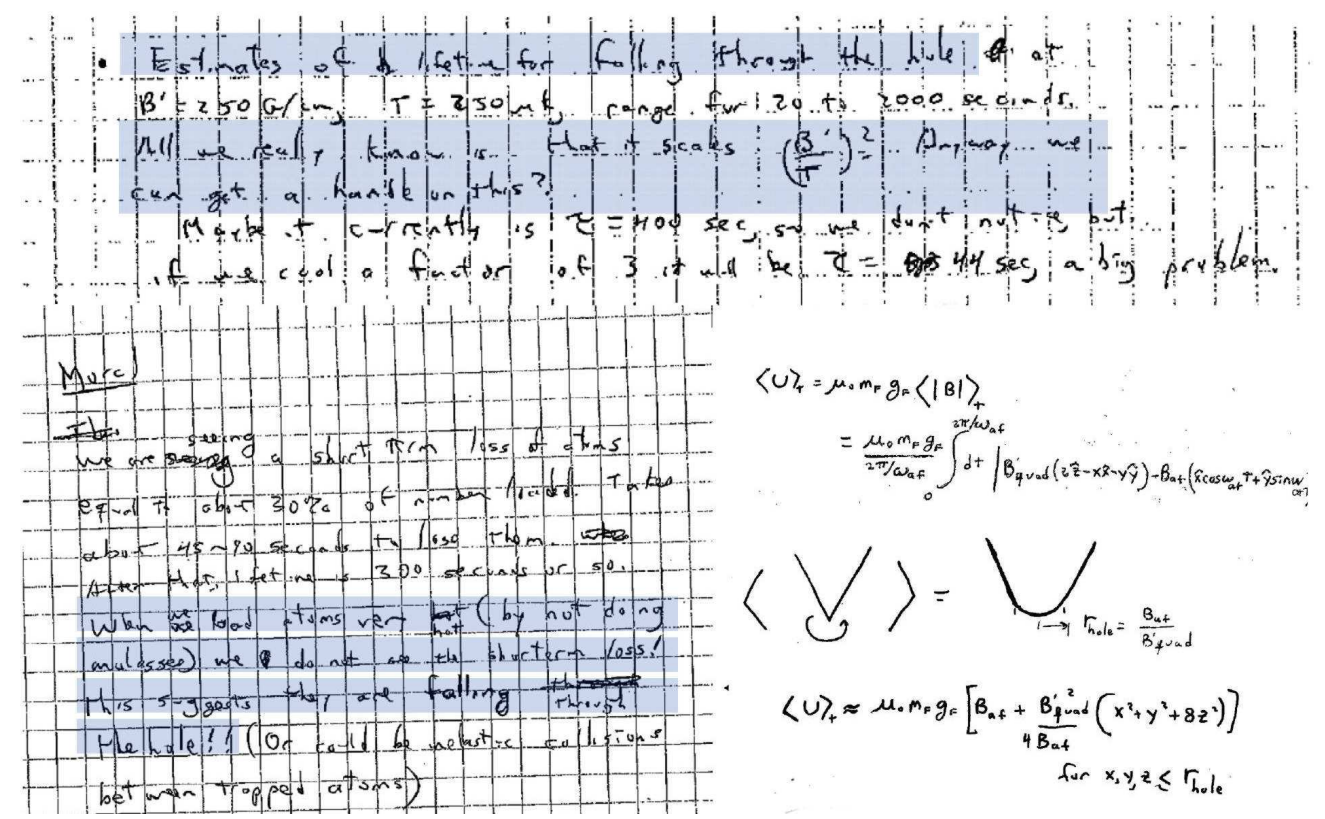

Figure 7: Hand-written notes of E. Cornell in the laboratory notebooks at JILA during the first experiments that led to Bose-Einstein condensation. The first experimental evidences of Majorana losses in the trapped samples are reported, together with the new trap design to overcome such problem.

a nonzero minimum. The first solution came from the Boulder group using a quite sophisticated trap where the position of the minimum quickly rotated faster that the atom motion (TOP trap) and the parameters were chosen using Majorana's quantitative expression for the spin-flip. It is interesting to report what Cornell and Wieman write: "This zero represents a hole in the trap, a site at which atoms can undergo Majorana transitions and thus escape from the trap. ...the TOP design worked well, and the samples were cooled far colder, in fact too cold...". Ketterle, on the other hand, solved the problem by using a focused laser beam to "plug the hole". Fig. 7 shows some pages from the laboratory notebooks of JILA, in which it is possible to distinguish the hand-writing of E. Cornell. In these notes there is an explicit reference to the observation of atom losses due to the "Majorana hole", together with the first ideas for the new trap design that would have solved this problem.

Nowadays the problem is completely understood and it is possible to use different static configurations in order to avoid the problem of Majorana losses. One common trap design is the one shown in Fig. $6 \mathrm{~b}$, in which the modulus of the magnetic field varies harmonically around a nonzero minimum, in the so-called "Ioffe-Pritchard configuration". This kind of trap is used by F. S. Cataliotti and his group in Catania, the hometown of Majorana, where ultracold atoms are trapped on a microfabricated chip. Fig. 8 shows the temporal behaviour of the number of atoms trapped at a temperature of a few microkelvin in a quadrupole trap (left) and in a Ioffe-Pritchard trap (right): as can be observed, by "plugging the hole" and suppressing Majorana spin-flip transitions, the atomic-trap lifetime is significantly enhanced [12].

Once the condensate is realized, spin-flip transitions, this time induced in a controlled fashion, can be used for further developments in the physics of ultra-low temperatures. Recently, X. Chen 

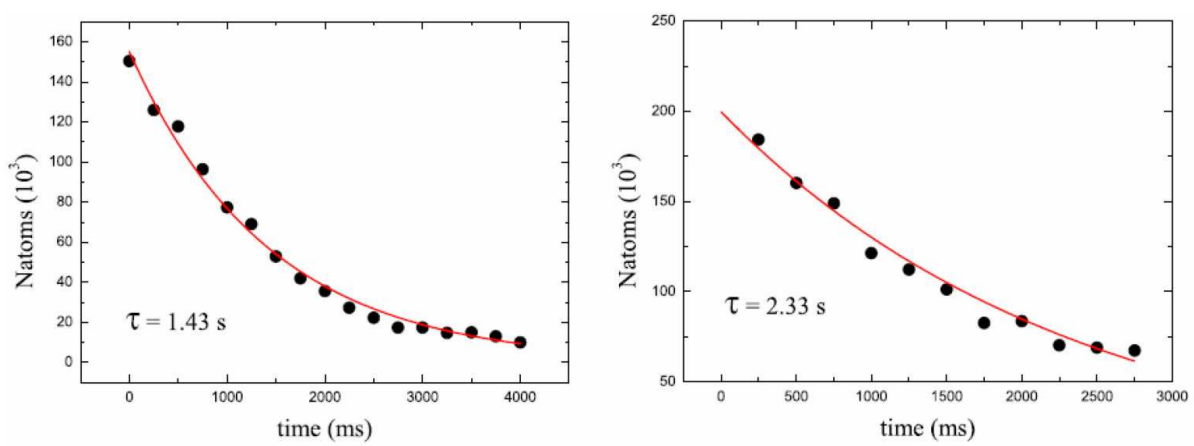

Figure 8: Atoms polarized and confined with magnetic field gradients on the route to Bose-Einstein condensation. The graph on the left refers to a quadrupole trap where there are losses due to Majorana spin-flip. The graph on the right refers to a trap specifically designed to avoid spin-flips. In both graphs the number of trapped atoms is plotted as a function of time [12].

in Beijing University [13] has used Majorana's theoretical predictions to develop a new method to extract coherent atoms from a condensate and realize a pulsed atom laser.

\section{Acknowledgments}

I would like to thank stimulating discussions with F. Bassani, C. Cohen-Tannoudji and E. Cornell. The latter is also acknowledged for providing unpublished material. A special thank to my coworker L. Fallani for his help in preparing the material for this presentation.

\section{References}

[1] E. Majorana, Nuovo Cimento 9, 43 (1932); see also the English translation recently published in Ettore Majorana, Scientific Papers, on occasion of the centenary of the birth, edited by G. F. Bassani and the Council of the Italian Physical Society, SIF-Springer 2006.

[2] E. Amaldi, Ettore Majorana, man and scientist, in Strong and Weak Interactions, Present Problems, International School of Physics Ettore Majorana, Erice, June 19th - July 4th 1966, edited by A. Zichichi, Academic Press, New York and London 1966.

[3] R. Frisch and E. Segré, Nuovo Cimento 10, 78 (1933); Z. Phys. 80, 610 (1933).

[4] P. Güttinger, Z. Phys. 73, 169 (1932).

[5] F. Bloch and I. I. Rabi, Rev. Mod. Phys. 17, 237 (1945).

[6] L. D. Landau and E. M. Lifshitz, Quantum Mechanics: Non-relativistic Theory, Nauka, Moscow 1974; Pergamon Press, Oxford 1977.

[7] F. Di Giacomo and E. E. Nikitin, Phys. Usp. 48, 515 (2005).

[8] J. Brossel and F. Bitter, Phys. Rev. 86, 308 (1952) 308; see also J. Brossel, Thesis, Faculté des Sciences de l'Université de Paris 1952.

[9] C. Cohen-Tannoudji, lessons available online at http://www.phys.ens.fr/cours/college-de-france/ 
[10] E. A. Cornell and C. E. Wieman, Rev. Mod. Phys. 74, 875 (2002).

[11] W. Ketterle, Rev. Mod. Phys. 74, 1173 (2002).

[12] Courtesy of F. S. Cataliotti, Università di Catania 2006.

[13] X. Ma et al., Phys. Rev. A 73, 013624 (2006). 